\title{
Efficacy and Safety of Human Mesenchymal Stromal Cells in Healing of Critical-Size Bone Defects in Immunodeficient Rats
}

\author{
R. PYTLÍK ${ }^{1}$, C. RENTSCH ${ }^{2}$, T. SOUKUP ${ }^{3}$, L. NOVOTNÝ ${ }^{4,5}$, B. RENTSCH ${ }^{2}$, \\ V. KANDEROVÁ ${ }^{6}$, H. RYCHTRMOCOVÁ ${ }^{1}$, M. KALMÁROVÁ ${ }^{1}$, D. STEHLÍK $^{7,8}$, \\ M. TRNĚNÝ ${ }^{1}$, O. SLANA $\breve{R}^{9}$
}

${ }^{1}$ First Department of Medicine, First Medical Faculty, Charles University, Prague and General University Hospital, Prague, Czech Republic, ${ }^{2}$ Translational Bone, Joint and Soft Tissue Research, Medical Faculty and University Hospital Carl Gustav Carus, Technische Universität Dresden, Dresden, Germany, ${ }^{3}$ Institute of Histology and Embryology, Medical Faculty Hradec Králové, Charles University, Hradec Králové, Czech Republic, ${ }^{4}$ Radioisotope Laboratory and Animal House, Medical Faculty Hradec Králové, Charles University, Hradec Králové, Czech Republic, ${ }^{5}$ Finn Pathologists, One Eyed Lane, Weybread, Norfolk, IP21 5TT, United Kingdom, ${ }^{6}$ CLIP - Childhood Leukaemia Investigation Prague, Department of Paediatric Haematology and Oncology, Second Medical Faculty, Charles University, Prague and Motol University Hospital, Prague, Prague, Czech Republic, ${ }^{7}$ Institute of Anatomy, First Medical Faculty, Charles University, Prague, Czech Republic, ${ }^{8}$ Department of Orthopedics, Second Medical Faculty, Charles University, and Motol University Hospital, Prague, Czech Republic, ${ }^{9}$ Institute of Pharmacology, First Medical Faculty, Charles University, Prague and General University Hospital, Prague, Czech Republic

Received May 17, 2016

Accepted June 22, 2016

On-line October 26, 2016

\section{Summary}

To evaluate the preclinical efficacy and safety of human mesenchymal stem cells (hMSC) rapidly expanded in growth medium for clinical use with human serum and recombinant growth factors, we conducted a controlled, randomized trial of plasma clots with hMSC vs. plasma clots only in critical segmental femoral defects in rnu/rnu immunodeficient rats. X-ray, microCT and histomorphometrical evaluation were performed at 8 and 16 weeks. MSC were obtained from healthy volunteers and patients with lymphoid malignancy. Human MSC survived in the defect for the entire duration of the trial. MSC from healthy volunteers, in contrast to hMSC from cancer patients, significantly improved bone healing at 8 , but not 16 weeks. However, at 16 weeks, hMSC significantly improved vasculogenesis in residual defect. We conclude that hMSC from healthy donors significantly contributed to the healing of bone defects at 8 weeks and to the vascularisation of residual connective tissue for up to 16 weeks. We found the administration of hMSC to be safe, as no adverse reaction to human cells at the site of implantation and no evidence of migration of hMSC to distant organs was detected.

\section{Key words}

Human mesenchymal stromal cells - Bone regeneration • Athymic rats • Treatment efficacy • Safety

\section{Corresponding author}

R. Pytlík, First Department of Medicine, General University Hospital and First Medical Faculty of Charles University, U nemocnice 2, 12808 Prague 2, Czech Republic. E-mail: pytlikr@seznam.cz

\section{Introduction}

The healing of large bone defects remains one of the principle challenges of modern orthopedics. The best material for the surgical treatment of large defects is autologous bone graft. However, the amount of material is limited and the risk of morbidity at the harvest site is not negligible (Banwart et al. 1995, Swan et al. 2006). Cadaverous bone grafts are another option, however, the potential for treatment failure is higher than for 
autologous grafts.

Human mesenchymal stromal cells (hMSC) were first isolated from the bone marrow by Fridenstein roughly 50 years ago (Fridenstein et al. 1966, Fridenstein et al. 1968), and their potential for differentiation to several lineages was shown by Pittenger et al in 1999. Similar cells can also be harvested from other tissues (dental pulp, adipose tissue, Wharton jelly and others - Jiang et al. 2002, Boquest et al. 2006, Shi and Gronthos 2003, Montanucci et al. 2011).

The main disadvantages of bone marrow-derived hMSC are their relative paucity in the bone marrow and the need for long-term cultivation with serial passaging. We developed a new method for the cultivation of hMSC from bone marrow mononuclear cells, which is faster and more effective than traditional cultivation in a medium supplemented by fetal calf serum. In this method, CellGro ${ }^{\mathrm{TM}}$ Medium for Hematopoietic Cells (Cellgenix, Germany) supplemented by $10 \%$ human serum and insulin, dexamethasone, ascorbic acid, EGF, PDGF-BB, FGF-2 and M-CSF is used, as published previously (Pytlík et al. 2009). This method results in a high number of MSCs in just two weeks and without the need of passaging. Cells obtained by this method fulfill consensus criteria for mesenchymal stromal cells (Dominici et al. 2006) and produce more and better mineralized tissue after heterotopic implantation in immunodeficient mice. Furthermore, hMSC cultivated by this method show greater expression of several genes connected with ossification and skeletal and bone development, namely ARID5B, CDH11, COL1A1, COL12A1, CTGF, IGFBP3 and $P R R X 2$ by gene expression profiling and qRT-PCR analysis (unpublished data).

Due to their low immunogenicity, mesenchymal stromal cells survive for some time in a xenogenic setting, however, an immune response eventually occurs and cells are rejected (Wang et al. 2007). Therefore, immunodeficient animals provide a better environment for preclinical experiments with hMSC. Athymic rnu/rnu rats are frequently used in experimental studies concerning the role of hMSC in bone healing. Several studies on hMSC in critical size femoral or calvarial defects in athymic rats have been performed (Peterson et al. 2005, Yoon et al. 2007, Cuomo et al. 2009, Liu et al. 2010, Chen et al. 2013, Suenaga et al. 2015), however, in all these studies, osteoconductive scaffolds were used and hMSC were frequently preconditioned in vitro or were genetically manipulated. Despite this, complete healing of the defect occurred only in a minority of experiments.
We conducted a randomized, controlled animal trial to evaluate the safety and efficacy of hMSC cultivated by our proposed method according to the requirements of European Medical Agency (EMA). Critical size segmental femoral bone defects in rnu-rnu rats were filled with human plasma clots either with hMSC (experimental groups) or without them (control group). As many candidates for reconstructive orthopedic surgery are cancer patients, approximately one half of the experimental cohort were patients with lymphoid malignancy (lymphoma group), the other part healthy volunteers (healthy group). For better temporal evaluation of bone healing, we further subdivided the experimental and control groups into shortterm ( 8 weeks) and long-term (16 weeks) cohorts. Safety was assessed by evaluating explanted femurs and parenchymatous organs for pathologies directly or indirectly attributable to hMSC.

\section{Methods}

\section{Experimental setting}

A total of 42 animals were randomly assigned to three 8-week cohorts (healthy, lymphoma, control) and to three similarly named 16-week cohorts.

Human mesenchymal stromal cell collection and cultivation

Human mesenchymal stromal cells were cultivated from bone marrow mononuclear cells (BMMC) from healthy volunteer donors and patients undergoing bone marrow examination for suspected or proven lymphoid malignancy. The study was approved by the local ethics committee and all donors provided signed informed consent according to the Helsinki declaration.

BMMC were obtained after the centrifugation of $10 \mathrm{ml}$ of bone marrow blood on Ficoll-Paque gradient media and processed as described previously Shortly, $2.5 \times 10^{6}$ BMMC were seeded in $75 \mathrm{~cm}^{2}$ plastic flasks, cultivated in CellGro ${ }^{\mathrm{TM}}$ for Hematopoietic Cells medium with $10 \%$ human serum and insulin, ascorbic acid phosphate, dexamethasone, EGF, PDGF-BB and FGF-2 (suppliers were the same as in Pytlík et al. 2009). Non-adherent cells were not removed, cultivation medium was not changed and supplements were added twice per week for a total of four doses.

After two weeks, non-adherent cells were washed away and MSCs were harvested by EDTATrypsin (Gibco Life Technologies, Grand Island, New York, USA) and counted in Burker chambers. Viability 
was tested with trypan blue. Aliquots of cells were frozen for later flow cytometric analyses.

\section{Preparation of plasma and plasma/hMSC clots}

A suspension of $360 \mu \mathrm{l}$ human plasma and $40 \mu \mathrm{l}$ calcium chloride (Sigma-Aldrich Czech Republic, Prague, Czech Republic) was seeded into 24 well plates and allowed to clot. For experimental groups, cells were added before recalcification (target number, $10 \times 10^{6} \mathrm{hMSC}$ for clot). In preliminary experiments, hMSCs were cultivated in plasma clots in vitro for one week; after this period, cells were alive and proliferating (Fig. 1A, B).

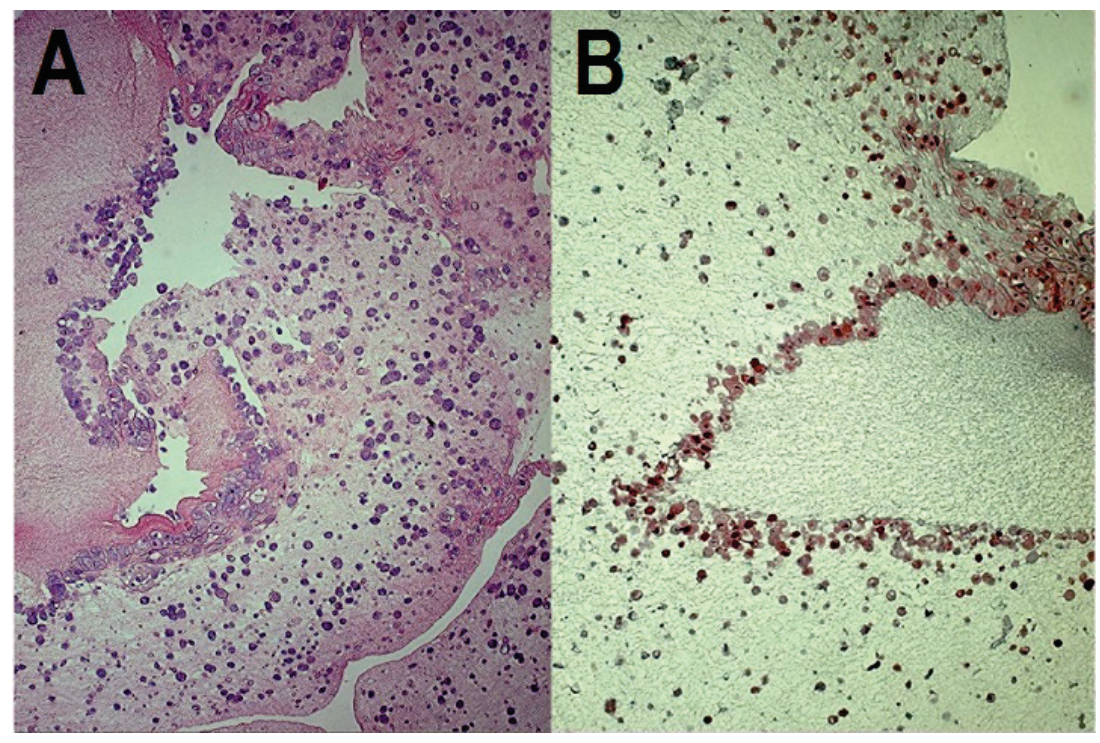

Fig. 1. Human $M S C$ in plasma clot (A) hematoxylin-eosin, (B) anti-Ki67 immunostaining.

Animal procedures

Athymic rnu/rnu rats (Charles River Laboratories, Sulzfeld, Germany) were kept in sterile cages and fed sterile food and water, until they reached skeletal maturity (10-14 weeks). Animals were cared for according to the European guidelines for the care and use of laboratory animals (Directive 24.11.1986, $86 / 609 / \mathrm{CEE})$. The experiment was approved by the Dresden regional veterinary board.

Rats were anesthetized with a solution of ketamine $35 \mathrm{mg} / \mathrm{kg}$ (Kemint, Alvetra $\mathrm{GmbH}$, Neumunster, Germany) and xylazine $5 \mathrm{mg} / \mathrm{kg}$ (Rompun, Bayer Healthcare Pharmaceuticals, Berlin, Germany). An incision was made along the left thigh and the diaphysis of the femur was dissected by blunt dissection. A 5-hole mini fragment plate (246190, Synthes, West Chester, PA, USA) was fixed to the femur using 2 screws (210.006, Synthes, Tuttlingen, Germany) above and below the proposed osteotomy site. Critical-sized defects (target $5 \mathrm{~mm}$ ) were created and plasma clots with or without cells were placed into the defects. Muscle tissue was folded back and closed with a resorbable suture and the skin was closed with a non-resorbable suture (Mariderm, Catgut GmbH, Markneukirchen, Germany).
Single-shot antibiotic prophylaxis with amoxicillin $15 \mathrm{mg} / \mathrm{kg}$ (Duphamox, Pfizer GmbH, Berlin, Germany) was administered and Carprofen $1.4 \mathrm{mg} / \mathrm{kg}$ (Rimadyl, Pfizer GmbH) was given immediately and $24 \mathrm{~h}$ after surgery for pain prevention. After 8 or 16 weeks, all animals were anesthetized (ketamine/xylazine mixture) and euthanized with a combination of embutramide, mebezonium and tetracaine (Tanax, Intervent $\mathrm{GmbH}$ Unterschleißheim, Germany).

\section{$X$-ray and microCT measurements}

$\mathrm{X}$-ray imaging of the defects was performed on an AMX4 machine (GE Healthcare, Buckinghamshire, UK) postoperatively (Fig. 2A) and 8 and 16 weeks after surgery. Defect size was evaluated using ImageJ software (imagej.nih.gov/ij). New bone formation (X-ray) was calculated as the postoperative defect minus residual defect (Fig. 2B). After sacrifice, microCT examination with 3D reconstructions were performed on vivaCT 75 (Scanco, Brüttisellen, Swizerland; Fig. 2C). Total volumes (TV) of tissue between two screws at both defect sites and new bone volumes (BV) were calculated in voxels. The ratio of bone volume to total volume (or bone volume fractional value) was calculated as BV/TV. 


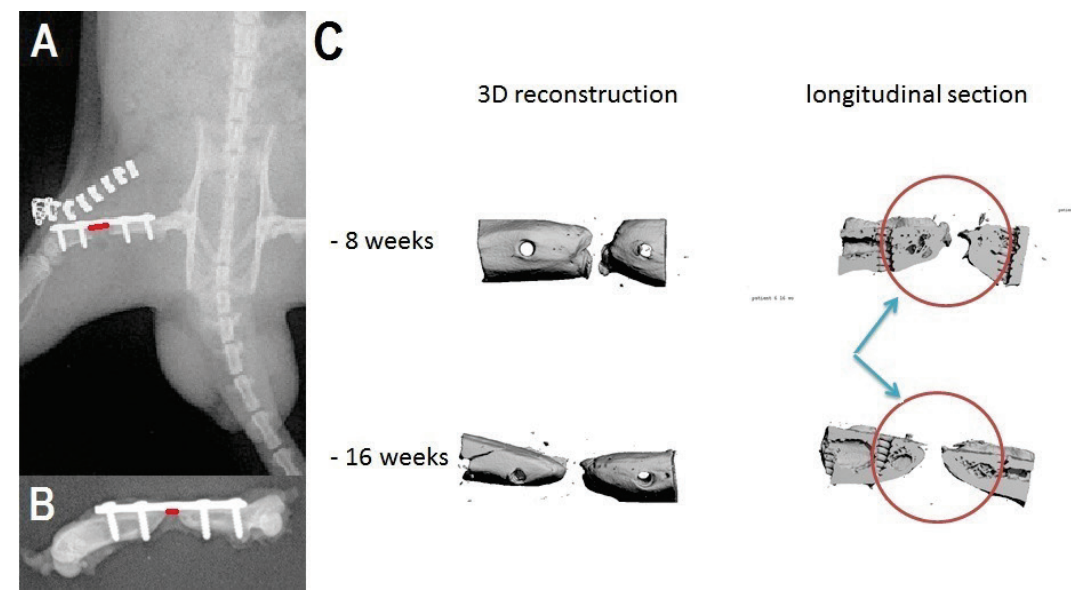

D

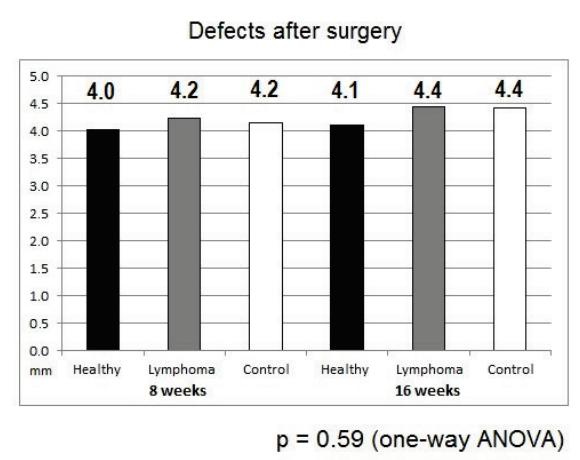

Fig. 2. (A, B) evaluation of defect size by X-ray, (C) evaluation of defect size by microCT, (D) defect size after surgery in experimental and control groups.

\section{Histological procedures}

Femoral explants were fixed in $4 \%$ buffered formalin (SAV LP, Flintsbach, Germany) for at least 7 days. The samples were washed, decalcified in ethylenediaminetetraacetic acid, dehydrated overnight and embedded in methyl methacrylate (Technovit 9100 New, Heraeus Kulzer GmbH, Werheim, Germany). Three- $\mu \mathrm{m}$ sections of each section plane were prepared, methylmethacrylate was removed and samples were rehydrated in a graded series of ethanol.

Femoral explants were stained with hematoxylin and eosin (Fig. 3A), Goldner (Fig. 3B, C) and Mallory stain. To prove the presence of human cells, anti-immunohistochemical tests for human nestin, clone 10C2 (Chemicon International, Inc., Temecula, CA, US). In several cases, immunofluorescent detection of human mitochondria, clone 235-1 (Genetex) was performed. Antigen retrieval was done in a citrate buffer solution using a microwave for $3 \times 5 \mathrm{~min}$. The standard $\mathrm{ABC}$ (Avidin Biotin Complex) method was used for the detection with DAB (3,3'-Diaminobenzidine), with Mason's green trichrome used as a counterstain (Fig. 3E). Fibroplasia found in one animal from the experimental group was further immunostained for vimentin, clone RV202 (Abcam, Cambridge, UK) and CD68, clone anti-ED1 (Abcam). Immunostaining for the evaluation of vessel formation was performed using actin detection, clone 1A4 (DAKO), visualized with $\mathrm{ABC}$ and fast red, with Mayer's hematoxylin as a counterstain (Fig. 3D).

Parenchymatous organs (liver, spleen, lungs, heart and kidneys) from each animal were fixed in $4 \%$ buffered formalin for 7 days. Fixed specimens were cut into tissue blocks embedded in paraffin and sectioned into slices $6-7 \mu \mathrm{m}$ thick. Sections were stained with hematoxylin and eosin and Masson's blue trichrome. Images were collected using an Olympus BX51 microscope equipped with a DP25 digital camera and were evaluated using ImageJ (FiJi, NIH). Histomorphometric quantification and vessel density in samples with residual fibrous tissue were tested in 10 samples from different areas (40 visual fields were observed).

\section{Flow cytometry analysis}

After thawing, cells were washed once in phosphate-buffered saline (PBS) supplemented with $0.5 \%(\mathrm{w} / \mathrm{v})$ bovine serum albumin (BSA, Carl Roth $\mathrm{GmbH}$, Karlsruhe, Germany). Aliquots of $1 \times 10^{5}$ cells were incubated with antibodies for $15 \mathrm{~min}$ at room temperature in the dark and washed again in PBS supplemented with $0.5 \%(\mathrm{w} / \mathrm{v})$ BSA. The data were collected with an LSR II or Canto II flow cytometer (BD Biosciences, San Jose, CA, USA). The following conjugated monoclonal antibodies were used: anti-CD3 APC, clone UCHT1 and anti-CD19 PE-Cy7, clone J3119, (both Beckman Coulter, Miami, FL, USA); anti-CD73 PE, clone AD2 and anti-CD90 APC, clone 5E10 (all Biolegend, San Diego, CA, USA); anti-CD16 PE, clone LNK16, anti-CD45 PerCP, clone MEM-28, anti-CD105 FITC clone MEM-226, and anti-CD235a FITC, clone HIR2 (all Exbio Praha, a.s., Vestec, Czech Republic). A minimum of 20,000 cells were acquired from each tube. The data were analyzed with FlowJo software (Treestar, Ashland, OR, USA). Cells were classified as hMSC according to CD105, CD90 and CD73 positivity, and CD45, CD19, CD235a, CD16 and CD3 negativity (Dominici et al. 2006). 

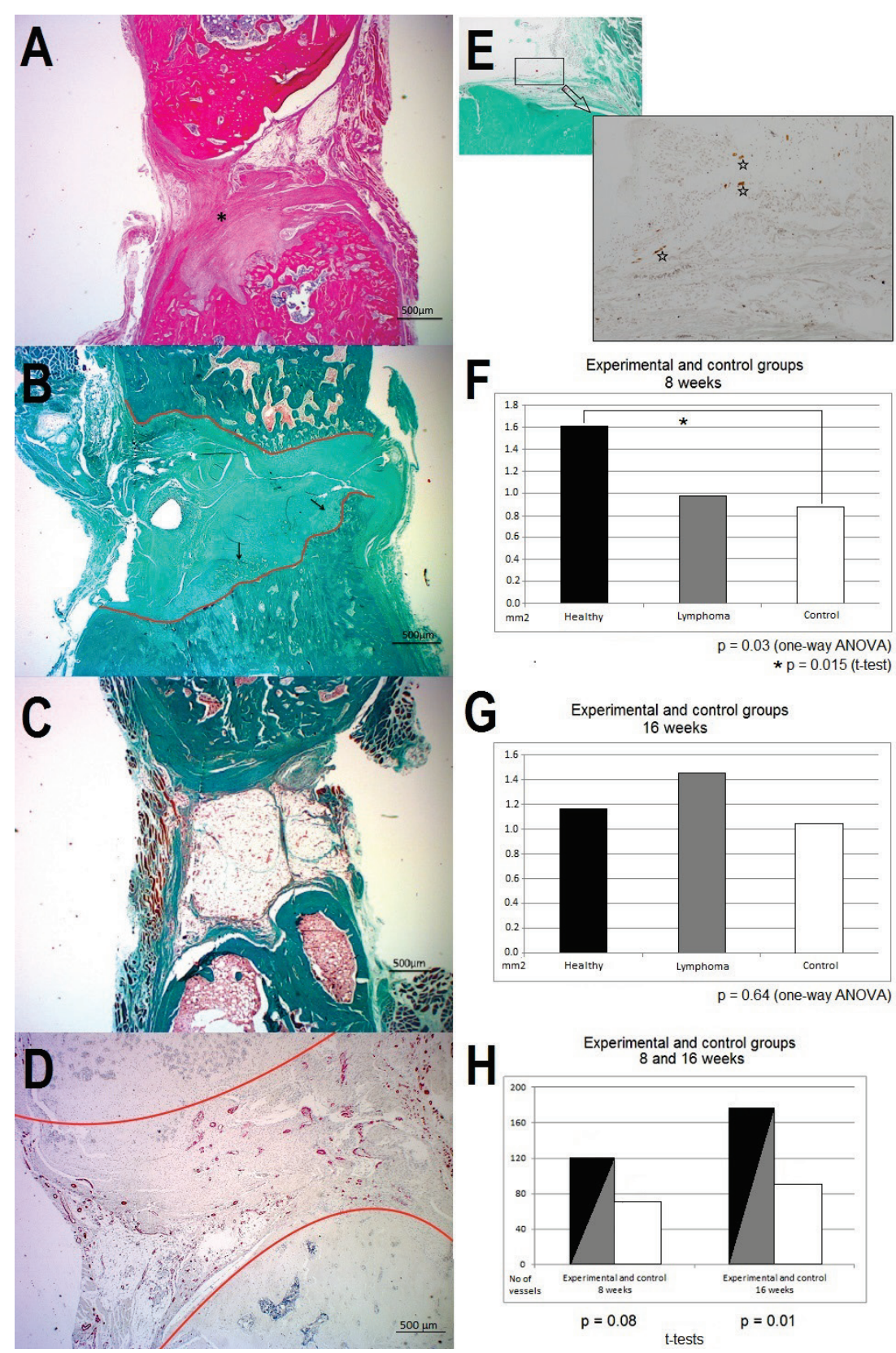

Fig. 3. Microscopy of bone healing and histomorphometric analyses. (A) Control No. 3, 8 week cohort: example of good healing with fibrous callus (asterisk) and residual adipose tissue (middle right). (B) Donor No. 12, 8 week cohort: example of good healing with fibrous callus, foci of chondroid differentiation (left arrow) and woven bone formation (right arrow). Lines for histomorphometrical evaluation are shown in red. (C) Control No. 4, 16 week cohort: example of poor healing with vascularized adipose tissue filling most of the defect. (D) Donor No. 3, 16 week cohort, staining for new vessels in fibrous callus (most of the defect) and adipose tissue (middle left). (E) Staining for human mitochondria. (F), (G) bone tissue formation by histomorphometry. (H) new vessel formation by histomorphometry. Experimental group means that healthy and lymphoma patients are counted together.

Stainings: (A) hematoxylin-eosin, (B, C) Goldner's trichrome, (D) anti-actin, fast red, Mayer's hematoxylin counterstain, (E) anti-human nestin, DAB, Mallory trichrome counterstain.
Statistics

Categorical variables were evaluated by chi-square tests. Continuous variables, in general, were normally distributed, therefore, parametric t-tests and parametric ANOVA were used for comparison among groups and cohorts, as appropriate. Paired t-tests were used for the comparison of paired samples. Correlations were calculated by the Pearson test. Flow cytometry data were not normally distributed, therefore, the MannWhitney U test was used for comparison between groups. Results are reported as mean \pm standard deviation, except for flow cytometry data where medians and ranges are reported. $p$ values $\leq 0.05$ were considered statistically significant. All calculations were performed with
STATISTICA software v. 12 (StatSoft, Tulsa, Minnesota, USA).

\section{Results}

\section{MSC donors and MSC cultivation}

Donor characteristics are shown in Table 1. In total, 13 donors were used (5 males and 8 females), with

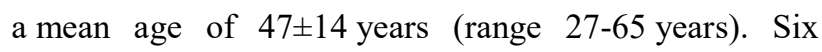
donors were patients with lymphoid malignancy, however, in only two cases bone marrow was infiltrated by lymphoma (1.4\% and $3.4 \%$ by FACS, respectively). Seven donors were healthy volunteers, significantly younger than lymphoma donors ( $38 \pm 11$ years vs. 
$55 \pm 10$ years, $p=0.008$ ). From all but one donor, there were enough cells for two clots and in 19 of 25 clots (76\%) the target number of $10 \times 10^{6}$ cells was implanted. The viability of implanted cells was consistently above
$90 \%$. The median number of cells CD105+CD90+CD73+ was 82.9\% (range 57.8-94.9\%), while the median CD45, CD235a, CD16, CD19 and CD3 positivities were $1.7 \%, 1.2 \%, 1.4 \%, 3.1 \%$ and $0.5 \%$.

Table 1. MSC donor characteristics.

\begin{tabular}{lccccccc}
\hline $\begin{array}{l}\text { Donor } \\
\text { number }\end{array}$ & Donor type & Sex & Age & $\begin{array}{c}\text { Experimental } \\
\text { group }\end{array}$ & Diagnosis & BM involvement & Cells implanted \\
\hline 1 & Patient & Male & 66 & 8 weeks & DLBCL & No & one clot $10 \times 10^{6}$ \\
2 & Patient & Male & 62 & $8+16$ weeks & FL & No & two clots a $10 \times 10^{6}$ \\
3 & Volunteer & Male & 47 & $8+16$ weeks & Healthy & No & two clots a $10 \times 10^{6}$ \\
4 & Patient & Female & 55 & $8+16$ weeks & FL & No & two clots a $8.75 \times 10^{6}$ \\
5 & Volunteer & Female & 31 & $8+16$ weeks & Healthy & No & two clots a $10 \times 10^{6}$ \\
6 & Volunteer & Female & 27 & $8+16$ weeks & Healthy & No & two clots a $10 \times 10^{6}$ \\
7 & Volunteer & Female & 52 & $8+16$ weeks & Healthy & No & two clots a $8 \times 10^{6}$ \\
8 & Patient & Female & 59 & $8+16$ weeks & FL & Yes & two clots a $10 \times 10^{6}$ \\
9 & Patient & Male & 39 & $8+16$ weeks & FL & No & two clots a $10 \times 10^{6}$ \\
10 & Patient & Male & 62 & $8+16$ weeks & FL & Yes & two clots a $10 \times 10^{6}$ \\
11 & Volunteer & Female & 51 & $8+16$ weeks & Healthy & No & two clots a $10 \times 10^{6}$ \\
12 & Volunteer & Female & 28 & $8+16$ weeks & Healthy & No & two clots a $10 \times 10^{6}$ \\
13 & Volunteer & Female & 32 & $8+16$ weeks & Healthy & No & two clots a $7.5 \times 10^{6}$ \\
\hline
\end{tabular}

Abbreviations: $\mathrm{FL}=$ follicular lymphoma, $\mathrm{DLBCL}=$ diffuse large $\mathrm{B}$-cell lymphoma.

\section{Surgical outcomes}

Surgical outcomes are shown in Table 2. Fourty of fourty-four animals could be evaluated (91\%). Two animals died during the surgical procedure (both in in the 16-week control group) and osteosynthesis failed in two animals (one 8-week lymphoma group, one in 16-week healthy group). In experimental groups, 9 paired samples $(8+16$ weeks from the same donor) were available for statistical analyses.

On postoperative $x$-ray images, the mean size of the defect was $4.2 \pm 0.5 \mathrm{~mm}$, with no differences between the six cohorts $(p=0.59$; Fig. $2 D)$.

\section{$X$-ray and microCT measurements}

8 and 16 weeks $x$-rays

In all six cohorts, some animals healed well and others less well (Fig. 4A-D). After 8 weeks, differences between three groups were not significant by ANOVA $(p=0.21)$, however, healthy group had significantly better healing than control group (new bone formation $2.7 \pm 1$ vs. $1.6 \pm 1 \mathrm{~mm}$ in the control group, $\mathrm{p}=0.05$, Fig. $2 \mathrm{E}$ ). There was some negative correlation between age and new bone formation, which was not statistically significant $(\mathrm{r}=-0.48, \mathrm{p}=0.19)$. After 16 weeks, there was no difference between healthy, lymphoma or control cohorts $(p=0.33)$ and neither healthy nor experimental group were better than controls (Fig. 4F).

\section{8- and 16-week microCT measurements}

MicroCT measurements correlated well with X-ray measurements $(\mathrm{r}=-0.73, \mathrm{p}=0.03)$, however, differences at 8 or 16 weeks were not statistically significant ( $p=0.29$ and 0.60 by ANOVA, respectively, Fig. 5A, B). Only a trend for higher BV/TV ratio in the healthy versus control group was observed at 8 weeks $(22 \pm 5 \%$ vs. $18 \pm 3 \%, \mathrm{p}=0.09)$.

\section{Histopathology}

Femoral explants, general histology

Good and poor defect bridgings were seen both in the experimental and control groups, and in both the 8 and 16 weeks cohorts (Fig. 3A-C). In cases of good bridging, a fibrous callus with foci of chondroid differentiation and woven bone formation was found (Fig. 3A, B). In cases of poor bridging, residual space 
was generally filled with adipose tissue with numerous blood vessels (Fig. 3C, D). Scattered human cells, detected by anti-human nestin, anti-human nuclei and anti-human mitochondrial antibodies were found in fibrous and adipose tissue in all examined cases (Fig. 3E), unfortunately, they could not be quantified due to technical reasons. No human cells were found in newly formed bone or cartilaginous tissue. No signs of inflammatory reaction or tumor formation were found.

Table 2. Surgical outcomes.

\begin{tabular}{|c|c|c|c|c|c|c|}
\hline & \multicolumn{6}{|c|}{ Groups } \\
\hline & healthy & $\begin{array}{c}\text { 8-week } \\
\text { lymphoma }\end{array}$ & control & healthy & $\begin{array}{l}\text { 16-week } \\
\text { lymphoma }\end{array}$ & control \\
\hline Animals operated & 7 & 6 & 9 & 7 & 5 & 10 \\
\hline Perioperative mortality & 0 & 0 & 0 & 0 & 0 & 2 \\
\hline Osteosynthesis failure & 0 & 1 & 0 & 1 & 0 & 0 \\
\hline Animals evaluated & 7 & 5 & 9 & 6 & 5 & 8 \\
\hline
\end{tabular}
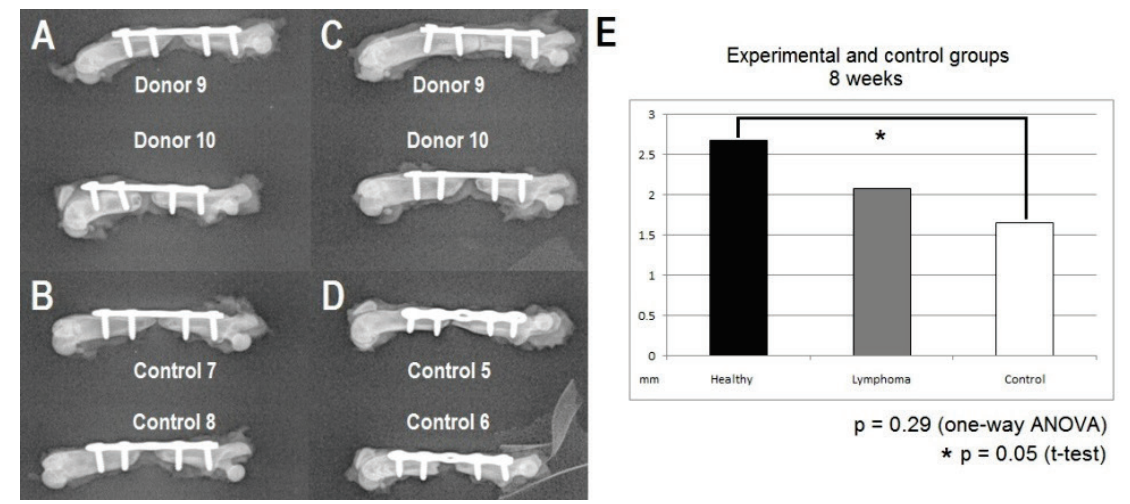

F

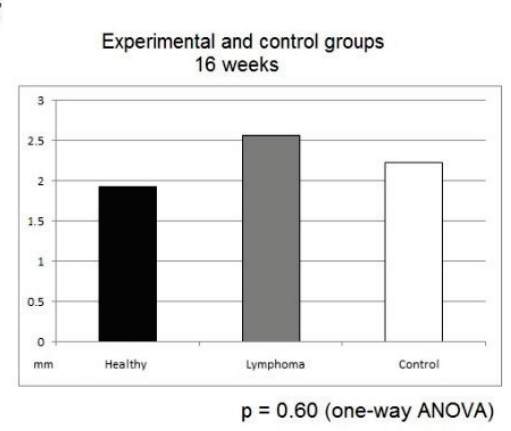

Fig. 4. (A-D) examples of good and poor healing in experimental and control groups as evaluated by $x$-ray: (A) 8-week experimental cohort, (B) 8-week control cohort, (C) 16-week experimental cohort, (D) 16-week control cohort; (E, F) comparison of new bone formation by $x$-ray measurements after 8 and 16 weeks in experimental and control groups and in healthy donor subgroups and control groups: (E) 8-week cohorts, (F) 16-week cohorts.

A

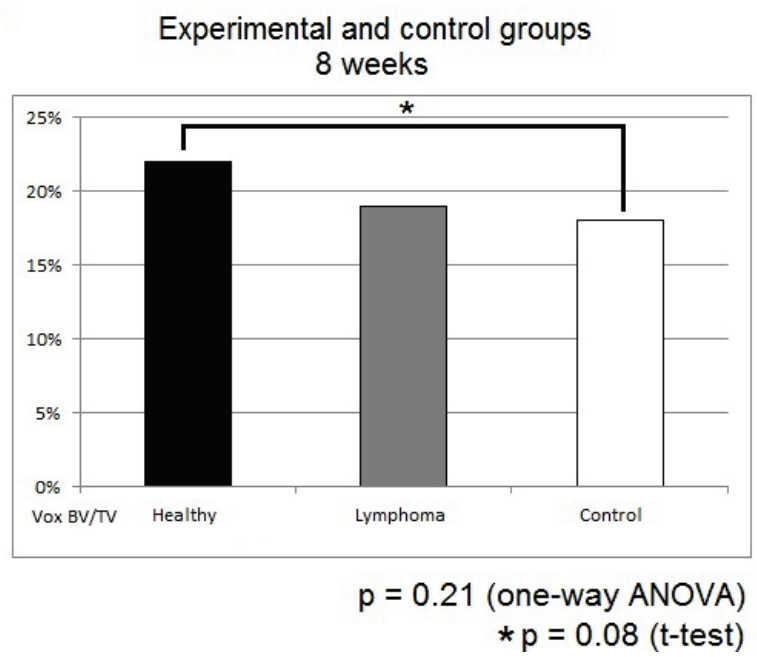

B

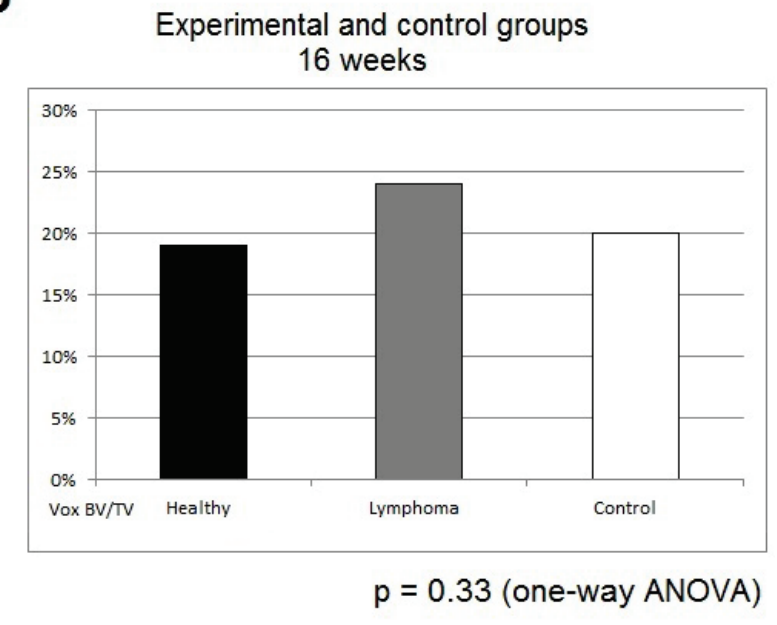

Fig. 5. Comparison of new bone formation expressed as bone volume fractional value (BV/TV) in microCT measurements in experimental and control groups and healthy donor subgroups and control groups: (A) 8-week cohorts, (B) 16-week cohorts. 


\section{Histomorphometry - new bone formation}

After 8 weeks, the amount of the new bone was significantly different between three groups $(p=0.03$ by ANOVA). Better bone formation was noted again for healthy vs. control group $\left(1.61 \pm 0.25\right.$ vs. $0.88 \pm 0.33 \mathrm{~mm}^{2}$, $\mathrm{p}=0.015$; Fig. $3 \mathrm{~F}$ ). After 16 weeks, no differences in bone formation were seen between the three groups $(p=0.64)$, Fig. 3G). Again, correlations between histomorphometry and new bone formation measured by x-ray and micro CT were strong and statistically significant (histomorphometry vs. X-ray, $\mathrm{r}=0.68, \mathrm{p}=0.0009$, histomorphometry vs. $\mathrm{BV} / \mathrm{TV}, \mathrm{r}=0.64, \mathrm{p}=0.002$ ).

\section{Histomorphometry - new vessel formation}

There was a trend for better vessel formation at 8 weeks in the experimental groups (121 \pm 46 vs. $71 \pm 26$ vessels, $\quad \mathrm{p}=0.07)$, which reached statistical significance at 16 weeks $(177 \pm 56$ vs. $92 \pm 13$ vessels, $\mathrm{p}=0.01$; Fig. $3 \mathrm{H}$ ). In these measurements, no differences between healthy and lymphoma samples were found.

\section{Histology of other organs}

In the myocardium of the left ventricle of animal No. 44 (Donor No. 13 in the 8-week cohort), we observed dense cellular proliferation of spindle-shaped to pleomorphic mesenchymal cells replacing cardiomyocytes. The cells were arranged in disorganized streams and bundles separated by a small to moderate amount of collagenous matrix, and had a low to moderate amount of pale eosinophilic cytoplasm and large oval vesicular nuclei with prominent medium-sized nucleoli. Anisocytosis and anisokaryosis were moderate. Immunohistochemical evaluation revealed vimentin positivity and CD68 negativity. The morphology, arrangement and immunohistochemistry of cells suggested reactive or reparative fibroplasia (Fig. 6). Cells of human origin were not detected here. In other animals, no microscopic changes and no evidence of human cells were found in parenchymatous organs.

\section{Discussion}

Cellular therapy and tissue engineering hold great promise for regenerative medicine and may change the future of clinical practice. Various materials with osteoconductive properties can be combined with osteoinductive factors and hMSC. These advanced therapy medicinal products (ATMP) must comply with

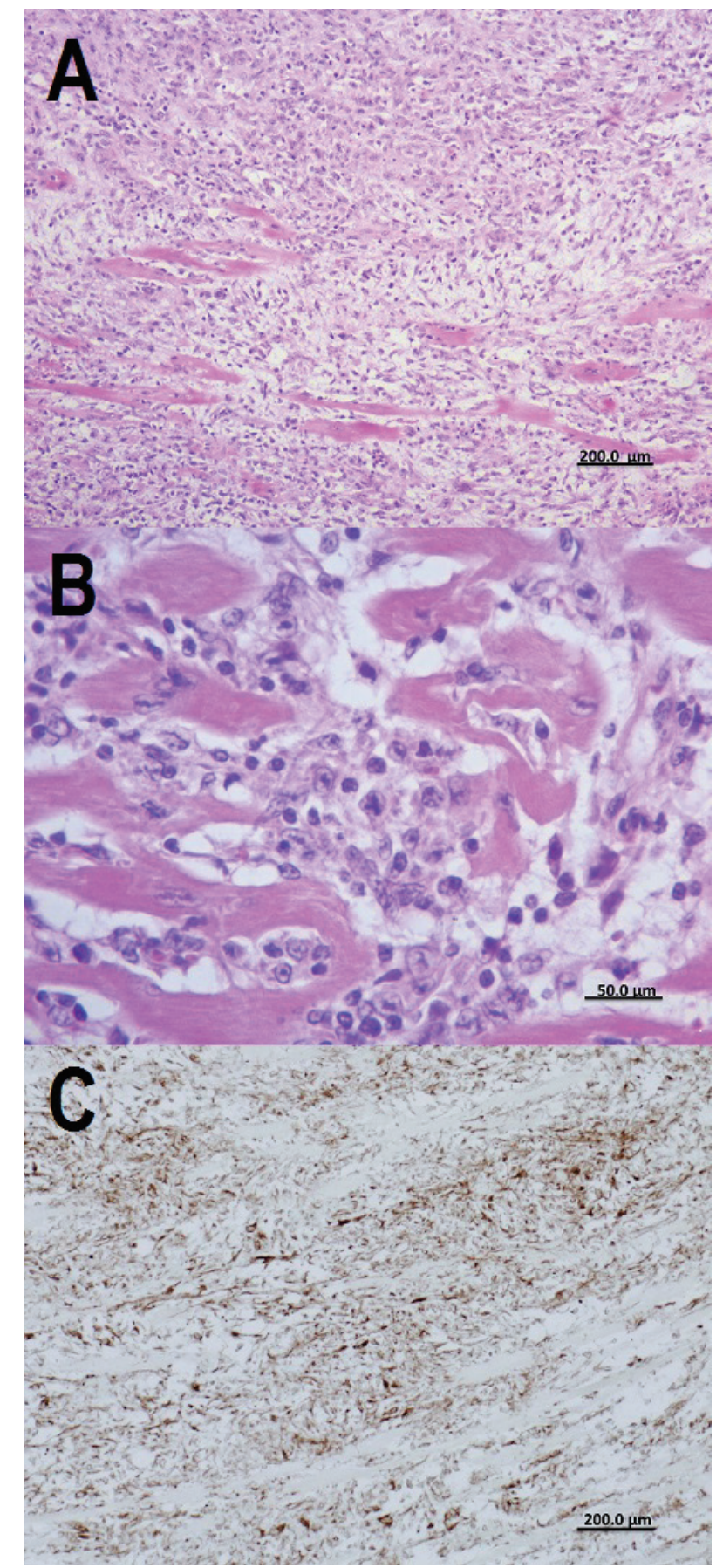

Fig. 6. Fibroplasia in left ventricular myocardium of animal no. 44, healthy donor No.13, 8-week experimental group. (A) hematoxylin-eosin, low magnification, (B) hematoxylin-eosin, high magnification, (C) anti-vimentin immunostaining, low magnification.

regulatory requirements including good manufacturing practice (GMP) and proof of product safety and efficacy. The more complex the product, the more complex the regulatory issues (Salmikangas et al. 2015).

Human MSC contribute to the healing of various organs both by direct and indirect mechanisms involving 
the production of cytokines and growth factors (Horwitz and Prater 2009). Furthermore, the immunomodulatory properties of hMSC inhibit inflammatory reactions in damaged tissues (Urdzíková et al. 2014).

Two basic models are used in bone regeneration experiments in rats: calvarial defects and segmental femoral defects. In calvarial defects, a punch-hole of $5-8 \mathrm{~mm}$ in diameter is considered to be of critical size. Yoon et al. (2007) used undifferentiated or in vitro osteogenically predifferentiated adipose tissue-derived stem cells (ATSC) on poly-lactic-co-glycolic-acid (PLGA) scaffolds. Fourteen day predifferentiated ATSC produced the best results, however, only $35-72 \%$ of the defect was healed. Chen et al. (2013) used bone marrow hMSC (BMMSC) and umbilical cord blood stem cells (UCBSC) with calcium phosphate and both improved healing compared to controls, however, even after 24 weeks, the newly formed bone covered no more than $35 \%$ of the defect in both hMSC groups. Also in the Suenaga et al. (2015) study, 3D-assembled hMSC (hMSC spheroids) combined with $\beta \mathrm{TCP}$ significantly, but only partially, contributed to the regenerative process.

The surgical creation of critical size femoral defects $(5-6 \mathrm{~mm})$ and their stabilization by internal fixation is likely a more physiologically relevant experimental model for bone healing. Cuomo et al. (2009) found bone marrow blood enriched with hMSCs seeded on demineralized bone tissue no better than demineralized bone tissue alone (1/10 defect healed at 12 weeks), while demineralized bone tissue or a collagen sponge with rhBMP-2 healed $6 \mathrm{~mm}$ defects effectively (9/10 defects healed). Liu et al. (2010) showed that human UCBSC in combination with partially demineralized bone matrix may completely heal critical femoral defects. In contrast, Peterson et al. (2005) showed that at 8 weeks, no healing occurred with adipose tissue-derived stem cells loaded on a collagen-ceramic carrier. These cells had to be transfected to express BMP-2 to fully heal critical-size defects. Altogether, published data are heterogenous, as various studies differ with regard to the source of hMSC, their predifferentiation or genetic modification, the type of scaffold, and the length of the experiment.

Our study differs from previous studies with regard to the method of hMSC preparation and lack of an osteoconductive scaffold. Rather, hMSCs were delivered in plasma clots, while plasma clots without hMSC formed the control group. As we have shown previously, hMSC grown in CellGro medium with human serum and supplements produced more mineralized tissue than hMSC grown with fetal calf serum, therefore, we did not consider necessary to use the latter as further control. Our experiment aimed at evaluation of performance of hMSC alone, without other supportive measures.

We observed significantly better bone healing with hMSC at eight weeks, but only in donors without malignant disease. Bone marrow-derived hMSC from patients with hematological malignancy have different functional properties than hMSC from healthy donors (Fei et al. 2014), and even may harbor karyotype abnormalities (Blau et al. 2011). Also lymphoma patients were older than healthy donor and, bone healing properties of hMSC may decrease with age (Stenderup et al. 2003). However, our cohort was too small for statistically meaningful measurement of interaction between age and health status of donors. In any case, hMSC were absent in newly formed bone and cartilaginous tissues. This is in agreement with the hypothesis that the role of hMSC in the regenerative processes is rather to deliver "software" (i.e. cytokines and growth factors) than "hardware" (direct contribution to healing).

After eight weeks, bone healing seemed to cease in both the control and experimental groups, but hMSC exerted further activity in the second eight weeks of the experiment, as significantly more vessels were found in the remaining connective tissue in the experimental groups compared to the control group. Therefore, we conclude that remodelling of the nonunion site is still going on and that with longer follow-up, the healing in experimental groups will continue.

The implantation of hMSC to the defect was safe. Human cells were not found in any of the examined parenchymatous organs. The only pathology found in sacrificed animals was one case of proliferation of rat mesenchymal cells in the myocardium, which is suggestive of reactive or reparative fibroplasia, which is a phenomenon well described in laboratory rats (Jokinen et al. 2011). No other pathologies, were found macroscopically or microscopically.

In conclusion, we have found that hMSC even without osteoconductive scaffold can contribute to bone healing in critical size femoral defect in immunodeficient rats. This contribution seemed to be limited to eight week but higher vascularisation in the experimental groups showed that remodelling of remaining tissue is still ongoing. To find out if smaller defect can heal with the hMSC contribution only while larger defects require 
some case of scaffold support, would need further experiments and with longer follow-up.

\section{Conflict of Interest}

There is no conflict of interest.

\section{Acknowledgements}

This work was supported exclusively by a grant from Ministry of Public Health, Czech republic, IGA NT 13531/4, 2011.

\section{References}

BANWART JC, ASHER MA, HASSANEIN RS: Iliac crest bone graft harvest donor site morbidity. A statistical evaluation. Spine 20: 1055-1060, 1995.

BLAU O, BALDUS CD, HOFMANN WK, THIEL G, NOLTE F, BURMEISTER T, TÜRKMEN S, BENLASFER O, SCHÜMANN E, SINDRAM A, MOLKENTIN M, MUNDLOS S, KEILHOLZ U, THIEL E, BLAU IW: Mesenchymal stromal cells of myelodysplastic syndrome and acute myeloid leukemia patients have distinct genetic abnormalities compared with leukemic blasts. Blood 118: 5583-5592, 2011.

BOQUEST AC, SHAHDADFAR A, BRINCHMANN JE, COLLAS P: Isolation of stromal stem cells from human adipose tissue. Methods Mol Biol 325: 35-46, 2006.

CHEN W, LIU J, MANUCHEHRABADI N, WEIR MD, ZHU Z, XU HH: Umbilical cord and bone marrow mesenchymal stem cell seeding on macroporous calcium phosphate for bone regeneration in rat cranial defects. Biomaterials 34: 9917-9925, 2013.

CUOMO AV, VIRK M, PETRIGLIANO F, MORGAN EF, LIEBERMAN JR: Mesenchymal stem cell concentration and bone repair: potential pitfalls from bench to bedside. J Bone Joint Surg Am 91: 1073-1083, 2009.

DOMINICI M, LE BLANC K, MUELLER I, SLAPER-CORTENBACH I, MARINI F, KRAUSE D, DEANS R, KEATING A, PROCKOP DJ, HORWITZ E: Minimal criteria for defining multipotent mesenchymal stromal cells. The International Society for Cellular Therapy position statement. Cytotherapy 8: 315-317, 2006.

FEI C, ZHAO Y, GU S, GUO J, ZHANG X, LI X, CHANG C: Impaired osteogenic differentiation of mesenchymal stem cells derived from bone marrow of patients with lower-risk myelodysplastic syndromes. Tumour Biol 35: 4307-4316, 2014.

FRIEDENSTEIN AJ, PIATETZKY SII, PETRAKOVA KV: Osteogenesis in transplants of bone marrow cells. J Embryol Exp Morphol 16: 381-390, 1966.

FRIEDENSTEIN AJ, PETRAKOVA KV, KUROLESOVA AI, FROLOVA GP: Heterotopic of bone marrow. Analysis of precursor cells for osteogenic and hematopoietic tissues. Transplantation 6: 230-247, 1968.

HORWITZ EM, PRATHER WR: Cytokines as the major mechanisms of mesenchymal stem cell clinical activity: expanding the spectrum of cell therapy. Isr Med Assoc J 11: 209-211, 2009.

JIANG Y, VAESSEN B, LENVIK T, BLACKSTAD M, REYES M, VERFAILLIE CM: Multipotent progenitor cells can be isolated from postnatal murine bone marrow, muscle, and brain. Exp Hematol 30: 896-904, 2002.

JOKINEN MP, LIEUALLEN WG, BOYLE MC, JOHNSON CL, MALARKEY DE, NYSKA A: Morphologic aspects of rodent cardiotoxicity in a retrospective evaluation of National Toxicology Program Studies. Toxicol Pathol 39: 850-860, 2011.

LIU G, LI Y, SUN J, ZHOU H, ZHANG W, CUI L, CAO Y: In vitro and in vivo evaluation of osteogenesis of human umbilical cord blood-derived mesenchymal stem cells on partially demineralized bone matrix. Tissue Eng Part A 16: 971-982, 2010.

MONTANUCCI P, BASTA G, PESCARA T, PENNONI I, DI GIOVANNI F, CALAFIORE R: New simple and rapid method for purification of mesenchymal stem cells from the human umbilical cord Wharton jelly. Tissue Eng Part A 17: 2651-2661, 2011.

PETERSON B, ZHANG J, IGLESIAS R, KABO M, HEDRICK M, BENHAIM P, LIEBERMAN JR: Healing of critically sized femoral defects, using genetically modified mesenchymal stem cells from human adipose tissue. Tissue Eng 11: 120-129, 2005. 
PITTENGER MF, MACKAY AM, BECK SC, JAISWAL RK, DOUGLAS R, MOSCA JD, MOORMAN MA, SIMONETTI DW, CRAIG S, MARSHAK DR: Multilineage potential of adult human mesenchymal stem cells. Science 284: 143-146, 1999.

PYTLÍK R, STEHLÍK D, SOUKUP T, KALBÁCOVÁ M, RYPÁCEK F, TRC T, MULINKOVÁ K, MICHNOVÁ P, KIDERYOVÁ L, ZIVNÝ J, KLENER P JR, VESELÁ R, TRNENÝ M, KLENER P: The cultivation of human multipotent mesenchymal stromal cells in clinical grade medium for bone tissue engineering. Biomaterials 30: 3415-3427, 2009.

SALMIKANGAS P, SCHUESSLER-LENZ M, RUIZ S, CELIS P, REISCHL I, MENEZES-FERREIRA M, FLORY E, RENNER M, FERRY N: Marketing Regulatory Oversight of Advanced Therapy Medicinal Products (ATMPs) in Europe: The EMA/CAT Perspective. Adv Exp Med Biol 871: 103-130, 2015.

SHI S, GRONTHOS S: Perivascular niche of postnatal mesenchymal stem cells in human bone marrow and dental pulp. J Bone Miner Res 18: 696-704, 2003.

STENDERUP K, JUSTESEN J, CLAUSEN C, KASSEM M: Aging is associated with decreased maximal life span and accelerated senescence of bone marrow stromal cells. Bone 33: 919-926, 2003.

SUENAGA H, FURUKAWA KS, SUZUKI Y, TAKATO T, USHIDA T: Bone regeneration in calvarial defects in a rat model by implantation of human bone marrow-derived mesenchymal stromal cells spheroids. J Mater Sci Mater Med 26: 254, 2015.

SWAN MC, GOODACRE TE: Morbidity at the iliac crest donor site following bone grafting of the cleft alveolus. Br J Oral Maxillofac Surg 44: 129-133, 2006.

URDZÍKOVÁ LM, RŮŽIČKA J, LABAGNARA M, KÁROVÁ K, KUBINOVÁ Š, JIRÁKOVÁ K, MURALI R, SYKOVÁ E, JHANWAR-UNIYAL M, JENDELOVÁ P. Human mesenchymal stem cells modulate inflammatory cytokines after spinal cord injury in rat. Int J Mol Sci 15: 11275-11293, 2014.

WANG Y, CHEN X, ARMSTRONG MA, LI G: Survival of bone marrow-derived mesenchymal stem cells in a xenotransplantation model. J Orthop Res 25: 926-932, 2007.

YOON E, DHAR S, CHUN DE, GHARIBJANIAN NA, EVANS GR: In vivo osteogenic potential of human adiposederived stem cells/poly lactide-co-glycolic acid constructs for bone regeneration in a rat critical-sized calvarial defect model. Tissue Eng 13: 619-627, 2007. 